CODE: 025

Topic: Rural buildings, facilities and territory

Type of presentation: Poster.

\title{
8.8 MILK-PRODUCTION IN BARNS WITH COMPOST BEDDING AND FREE STALL: A PROFITABILITY ANALYSIS
}

\author{
Marcos Aurélio Lopes ${ }^{1 *}$, Gustavo Rafael de Oliveira Silva ${ }^{1}$; André Luís Ribeiro Lima ${ }^{1}$; Geraldo \\ Márcio da Costa ${ }^{1}$; Flávio Alves Damasceno ${ }^{1}$; Vitor Pires Barros ${ }^{2}$; Matteo Barbari ${ }^{3}$ \\ ${ }^{1}$ Federal University of Lavras, Campus Universitário, PO Box 3037, Lavras, Minas Gerais, \\ Brazil,37200-000,Fax: 55353829 1148.E-mail:malopes@ufla.br \\ ${ }^{2}$ Recursos Humanos no Agronegócio - Rehagro \\ ${ }^{3}$ Department of Agriculture, Food, Enveroment and Forestry (DAGRI), Università degli Studi di \\ Firenze. \\ * Author for correspondence
}

\begin{abstract}
:
Nowadays two intensive systems are used to produce milk from dairy cows: the free stall system and the compost barn system. The latter only recently has been introduced into several Countries, including Brazil. Various studies have addressed the dimensions, installation cost, and parameters to be measured in a compost barn, and other works cite possible advantages of the system in relation to reproduction, mastitis, and hoof injuries. However, no study has reported a profitability analysis of compost barn systems in the world. The objective of this study was to comparatively analyse the profitability of compost barn and free stall milk-production systems in Brazil as a mean of helping producers and technicians to choose the type of facility most suitable for each farm. Data collected from four farms from January to December 2016 were analysed. The data were distributed equally among compost barn and free stall systems. The cost of milk production was estimated according to the operating cost methodology and considering the milk production cost centre, which involved both lactating and dry cows. Additionally, gross and net margins were estimated as indicators of profitability. The results showed that the average gross and net margins were not influenced by the type of facility. They were positive in both of the production systems analysed, indicating that they are able to produce in the short and medium term. By comparing the components of the total operating cost of the compost barn and free stall farms, it was concluded that there were differences only in the "medications" item, which constituted a lower proportion of the compost barn farms due to the lower percentage expended for intramammary antibiotics for mastitis, and in the "bedding for cows" item, which represented the lowest proportion in the free stall system. Depreciation and total operating cost were similar in the two systems. Milk sales made up a higher percentage of the revenue in the free stall farms, while the expectations of revenues from wastes were similar in the two production systems. Given that there were no significant economic differences between the types of facility, it is concluded that ease in management, productivity, reproductive performance, animal health (hoof injuries and mastitis), environmental issues, and availability of water and bedding material should be the motivators for choosing one system over the other.
\end{abstract}

Key words: Dairy cattle, Compost bedding, Free stall, Cost centers, Production cost. 